\title{
Trasplante hepático en pacientes con trombosis de la vena porta*
}

\author{
Drs. MAURICIO GABRIELLI N. ${ }^{1}$, JOSÉ LUIS GALINDO R. ${ }^{1}$, EDUARDO FIGUEROA R. ${ }^{1}$, \\ FABRIZIO MOISAN P. ${ }^{1}$, MARCO ARRESE J. ${ }^{2,3}$, CARLOS BENÍTEZ G. ${ }^{2,3}$, \\ ALEJANDRO SOZA R. ${ }^{2,3}$, E.U. PILAR DOMÍNGUEZ B. ${ }^{3}$, ROSA MARÍA PÉREZ A. ${ }^{2,3}$, \\ JUAN FRANCISCO GUERRA C. ${ }^{1,3}$, NICOLÁS JARUFE C. ${ }^{1,3}$, JORGE MARTÍNEZ C. ${ }^{1,3}$
}

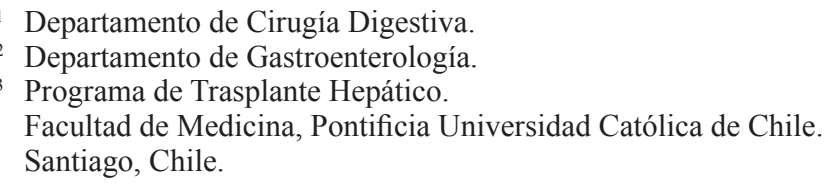

\section{Liver transplantation in patients with portal vein thrombosis}

Portal vein thrombosis (PVT) is a serious complication of end-stage liver disease, representing a challenge for the liver transplantation (LT) team. The aim of this study was to analyze the results of LT in patients with PVT. Methods: All adult patients who underwent a LT from a cadaveric donor between March 1994 and March 2010 were included. PVT was categorized using the classification of Yerdel and McMaster. Results: A total of 109 LT were performed in 105 patients. PVT was found in 13 cases (12.4\%). In 6 of them (46.2\%), thrombosis was found preoperatively by transplant routine work up. Pre-transplant systemic anticoagulation was indicated in 3 cases. At the time of surgery, only 10 patients had persistent PVT. Grade I, II, III and IV PVT was found on 2, 3, 4 and 1 patient respectively. In the 3 cases treated with systemic anticoagulation preoperatively, grade I ( 1 case) and grade II ( 2 cases) no thrombus was evident intraoperatively. Endovenectomy was performed in 7 cases; simple thrombectomy in one and a mesenteric vein graft was required in 2 cases. No thrombus recurrence was detected on postoperative follow-up. In-hospital mortality occurred in 2 cases with PTV grade III and IV due to medical complications. One-year patient and graft survival was $69 \%$ vs $79 \%$ in patients without PVT $(p=0,476)$. Conclusion: Liver transplant in patients with PVT thrombosis was not associated with a significant increased risk of mortality. Systemic anticoagulation seems to be warranted while waiting for liver transplantation.

Key words: Liver transplantation, portal vein thrombosis.

\section{Resumen}

La trombosis de la vena porta (TP) es una complicación grave de la enfermedad hepática crónica terminal. La factibilidad y pronóstico del trasplante hepático $(\mathrm{TH})$ dependen de su extensión en el eje porto-

*Recibido el 3 de septiembre de 2012 y aceptado para publicación el 8 de octubre de 2012.

Los autores no refieren conflictos de interés.

Correspondencia: Dr. Jorge Martínez C.

Marcoleta 352, Santiago, Chile. Fax: 56-2-638-2793

jamartin@med.puc.cl 
mesentérico. Objetivo: Analizar los resultados del trasplante hepático en pacientes con TP. Material y Método: Estudio de cohorte no concurrente a partir de los registros clínicos de los pacientes adultos con TP sometidos a un TH entre marzo de 1994 y marzo de 2010. Las TP fueron clasificadas en cuatro grados según Yerdel y Mc Master. Resultados: De 109 TH en 105 pacientes, 13 (12,4\%) tuvieron una TP; en 6 de ellos el diagnóstico fue preoperatorio (46,2\%). En tres se indicó tratamiento anticoagulante vía sistémica (TACO). En el intraoperatorio se encontró TP en 10 pacientes. La trombosis fue grado I, II, III y IV en 2, 3, 4 y 1 pacientes, respectivamente. Los 3 casos tratados con TACO tenían trombosis grado I y II; en ellos no se encontró trombosis durante el trasplante. Se realizó trombectomía simple en 1 paciente, trombectomía por eversión en 7 y un puente mesentérico-portal en 2 casos. No hubo re-trombosis. Dos pacientes $(15,4 \%)$ fallecieron de complicaciones médicas con injerto funcionante. La sobrevida al año de seguimiento año fue de un $69 \%$ vs $79 \%$ en los $\sin$ TP $(p=0,476)$. Conclusiones: El TH en pacientes con TP no se asoció a mayor mortalidad en relación a los pacientes trasplantados sin TP. El tratamiento anticoagulante en pacientes con TP puede indicarse mientras se espera el trasplante.

Palabras clave: Trasplante hepático, trombosis venosa portal.

\section{Introducción}

El trasplante hepático $(\mathrm{TH})$ ha revolucionado el cuidado de los pacientes con enfermedad hepática y se ha establecido como un tratamiento efectivo de la enfermedad hepática terminal de distintas causas ${ }^{1-4}$. En nuestro país, sus indicaciones han sido recientemente consensuadas y sus resultados son comparables a los de grandes centros internacionales ${ }^{5}$.

Con los avances en la técnica quirúrgica y los cuidados postoperatorios, las contraindicaciones para el trasplante hepático han disminuido ${ }^{6,7}$. La trombosis venosa portal (TP), considerada tradicionalmente una contraindicación para el trasplante ${ }^{8}$, es hoy aceptada como un factor más de riesgo de complicaciones intra y postoperatorias, pero susceptible de ser tratada previo o durante el trasplante. Se ha asociado a un mayor riesgo de sangrado, incrementando la complejidad del trasplante, su morbilidad y mortalidad $^{8-11}$.

La prevalencia comunicada de TP en la población general es de aproximadamente un $1 \%{ }^{12}$. Se estima que la incidencia de TP entre los pacientes con daño hepático crónico candidatos a $\mathrm{TH}$ varía entre un $13,8 \%$ y $38,9 \%{ }^{13}$.

El objetivo de este trabajo es analizar los resultados quirúrgicos y sobrevida en pacientes con TP sometidos a $\mathrm{TH}$.

\section{Material y Método}

Estudio de cohorte no concurrente a partir de la base de datos y registros clínicos de los pacientes adultos con TP sometidos a TH en nuestro centro entre marzo de 1994 y marzo de 2010. El estudio imagenológico de los pacientes durante los controles clínicos pre trasplante se realizó con ultrasonografía abdominal y resonancia magnética de abdomen. Las TP fueron clasificadas según su extensión en 4 grados de acuerdo a Yerdel y Mc Master ${ }^{14}$ : grado I: trombosis parcial $<50 \%$ con o sin extensión mínima a vena mesentérica superior (VMS); grado II: trombosis $>50 \%$ con o sin extensión mínima a VMS; grado III: TP y de VMS proximal completa, VMS distal libre y grado IV: TP y de VMS proximal y distal completa (Tabla 1). La técnica quirúrgica en el donante y en el receptor han sido previamente publicadas $^{15-17}$. Se realizó la hepatectomía en el receptor preservando la Vena Cava Inferior, con anastómosis cavo-cava, látero-lateral con técnica de Piggy-Back modificada ${ }^{15,17}$. Las anastómosis de la vena porta, de la arteria hepática y de la vía biliar fueron realizadas de preferencia en forma término-terminal.

Se analizó el momento del diagnóstico de la TP, el tratamiento preoperatorio utilizado, la técnica quirúrgica empleada, la tasa de re-trombosis, la falla del injerto con re-trasplante, y la morbilidad y mortalidad asociadas. Las complicaciones se clasificaron en precoces $(<$ de 3 meses) y tardías de acuerdo a las recomendaciones de la Sociedad Europea de Trasplantes (ESOT) y según gravedad, de acuerdo a la Clasificacion de Dindo y Clavien ${ }^{18,19}$ (Tabla 2).

\section{Resultados}

En el período descrito se realizaron $109 \mathrm{TH}$ en 105 pacientes adultos. De éstos, se diagnosticó una

Tabla 1. Clasificación de Yerdel y Mc Master para la trombosis portal*

\begin{tabular}{|lcl|}
\hline & Extensión & $\begin{array}{l}\text { Compromiso vena } \\
\text { mesentérica superior }\end{array}$ \\
\hline Grado I & $<50 \%$ & \pm obstrucción mínima \\
\hline Grado II & $50-100 \%$ & \pm obstrucción mínima \\
\hline Grado III & $100 \%$ & Proximal \\
Grado IV & $100 \%$ & Distal \\
\hline
\end{tabular}

*Transplantation 2000; 69: 1873-81. 
Tabla 2. Clasificación de Dindo y Clavien para las complicaciones postoperatorias*

\begin{tabular}{|c|c|}
\hline Grado & Definición \\
\hline \multirow[t]{2}{*}{ I } & $\begin{array}{l}\text { Desviación del postoperatorio normal, que no requiere de tratamiento farmacológico, quirúrgico, endoscópico o } \\
\text { intervenciones radiológicas }\end{array}$ \\
\hline & Fármacos permitidos: antieméticos, antipiréticos, analgésicos, diuréticos, electrolitos y fisioterapia \\
\hline \multirow[t]{2}{*}{ II } & Requerimiento de tratamiento farmacológico con drogas diferentes a las permitidas en las complicaciones grado I \\
\hline & Incluye transfusiones y nutrición parenteral \\
\hline III & Complicación que requiere una intervención quirúrgica, endoscópica o radiológica \\
\hline IIIa & No requiere anestesia general \\
\hline $\mathrm{IIIb}$ & Requiere anestesia general \\
\hline IV & Complicación que amenaza la vida del paciente y que requiere de cuidados intensivos \\
\hline IVa & Disfunción de un órgano (incluye diálisis) \\
\hline $\mathrm{IVb}$ & Falla multiorgánica \\
\hline $\mathrm{V}$ & Muerte del paciente \\
\hline
\end{tabular}

*Ann Surg. 2004; 240: 205-13.

Tabla 3. Características perioperatorias de los pacientes con trombosis portal

\begin{tabular}{|lcc|}
\hline Variable & & Pacientes $(\mathbf{n}=\mathbf{1 4})$ \\
Edad (años) & & $57[31-65]^{*}$ \\
Sexo & $\mathrm{F}$ & 5 \\
& $\mathrm{M}$ & 8 \\
Child-Pugh & $\mathrm{B}$ & 7 \\
& $\mathrm{C}$ & 6 \\
Tiempo operatorio (min) & $422[240-720]^{*}$ \\
Días UCI & $2,5[1-22]^{*}$ \\
Días postoperatorios & $17[8-29]^{*}$ \\
\hline
\end{tabular}

*Valores expresados en medianas y rangos.

Tabla 4. Etiología de la cirrosis hepática en los pacientes con trombosis portal

\begin{tabular}{|ll|}
\hline Etiología & n \\
Virus Hepatitis C & 4 \\
Esteatohepatitis no alcohólica & 3 \\
Alcohol & 2 \\
Hemocromatosis & 1 \\
Autoinmune & 1 \\
Criptogénica & 1 \\
Cirrosis Biliar Primaria & 1 \\
\hline
\end{tabular}

TP en $13(12,4 \%)$, con o sin extensión a la VMS. La mediana de edad de estos pacientes fue de 55 años [29-64]. Ocho $(61,5 \%)$ de sexo masculino. La función hepática pretrasplante de acuerdo a la clasificación de Child-Pugh fue $\mathrm{B}$ en 7 pacientes y $\mathrm{C}$ en los 6 pacientes restantes (Tabla 3 ). Las etiologías más frecuentes de la cirrosis hepática en los pacientes con TP fue el virus de la hepatitis C, la esteatohepatitis no alcohólica y el etanol (Tabla 4).

Se objetivó el diagnóstico de TP en el preoperatorio en 6 pacientes $(46,2 \%)$. De éstos, tres presentaron una TP diagnosticada precozmente y recibieron tratamiento anticoagulante (TACO) por vía sistémica, lográndose la resolución de la TP en todos ellos, de acuerdo a los hallazgos durante el trasplante. Así, en la intervención se confirmó una TP en 10 pacientes. La extensión de la TP según la clasificación de Yerdel y Mc Master ${ }^{14}$, se muestra en la Tabla 5.

En los pacientes con TP en el intraoperatorio, se realizó 1 trombectomía simple y 7 trombectomías

Tabla 5. Extensión de la trombosis portal según Yerdel, en los pacientes sometidos a trasplante hepático

\begin{tabular}{|c|c|}
\hline Grado & $\mathbf{n}$ \\
\hline I & 2 \\
II & 3 \\
III & 4 \\
IV & 1 \\
\hline
\end{tabular}




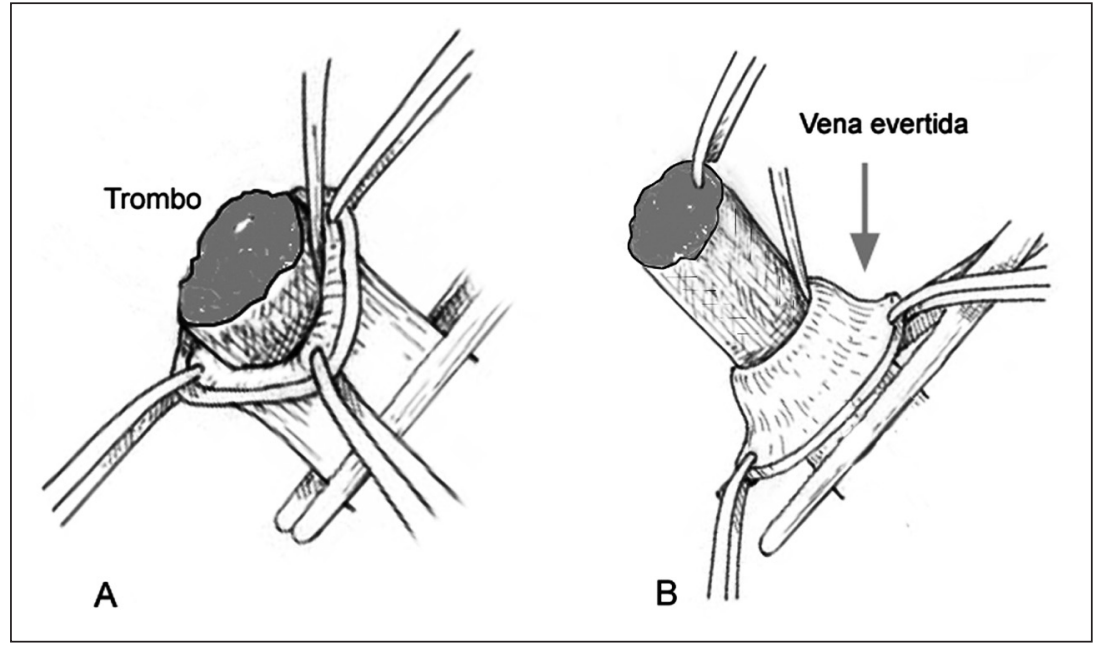

Figura 1. Trombectomía por eversión. A. Trombo en la vena porta. B. Eversión de la vena porta, con exposición completa del trombo. por eversión (Figuras 1 y 2). En dos casos fue necesario realizar una interposición de vena ilíaca del donante entre la vena mesentérica del receptor y la vena porta del injerto.

La presencia de TP no aumentó significativamente el tiempo operatorio del trasplante (mediana 422 [240-720] min vs 462 [160-840] min NS), los días de internación en la Unidad de Tratamiento Intensivo (mediana 2,5 [1-22] días vs 4 [1-88] días, NS) ni la duración de la estadía hospitalaria (mediana 17 [8-29] días, vs 17 [1-92] días, NS), al compararlos con los pacientes trasplantados sin TP.

Siete de 13 pacientes $(53,8 \%)$ presentaron una complicación precoz. Dos correspondieron a complicaciones tipo II de Clavien ${ }^{18,19}$, tres a complicaciones tipo III y dos a complicaciones tipo V, con fallecimiento de los pacientes (mortalidad 15,4\%). Los casos que no requirieron cirugía corresponden a un rechazo agudo severo grado III de la escala de Banff y a un infarto hepático segmentario.

Los tres pacientes que requirieron reoperación correspondieron a una trombosis de la arteria hepática, a una sospecha de peritonitis postoperatoria, que se descartó en el acto quirúrgico y a un hemoperitoneo que fue necesario explorar en dos oportunidades.

De los pacientes que fallecieron, el primer caso se debió a una hemorragia intracerebral asociada al tratamiento anticoagulante indicado por el puente venoso (día 21 postrasplante), en el contexto de una sepsis de foco pulmonar por neumonía asociada a ventilación mecánica. El segundo paciente falleció de una sepsis por Aspergillus el día 29 postrasplante.

Tres pacientes presentaron complicaciones tardías. Uno de ellos desarrolló una trombosis de la arteria hepática al año de evolución, con una biliopatía isquémica secundaria que requirió un retrasplante. Otro paciente desarrolló una estenosis de

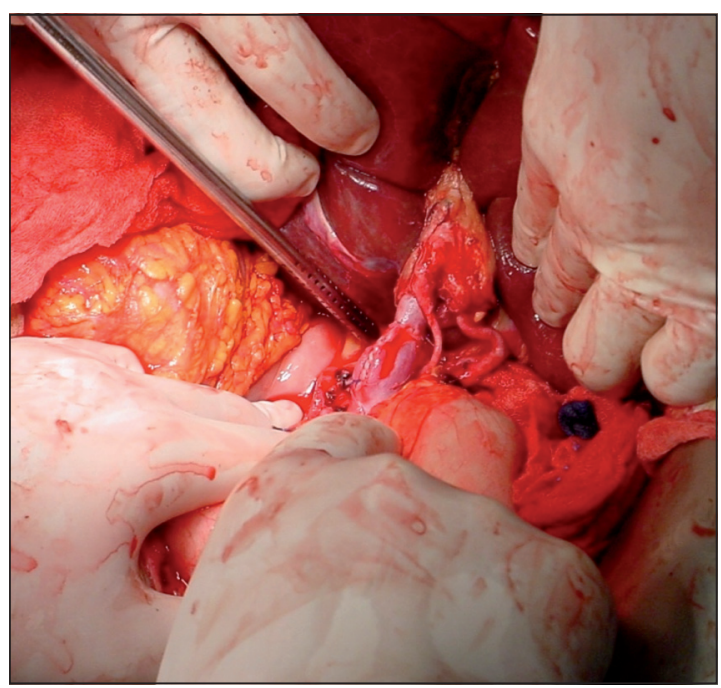

Figura 2. Anastomosis portal término-terminal.

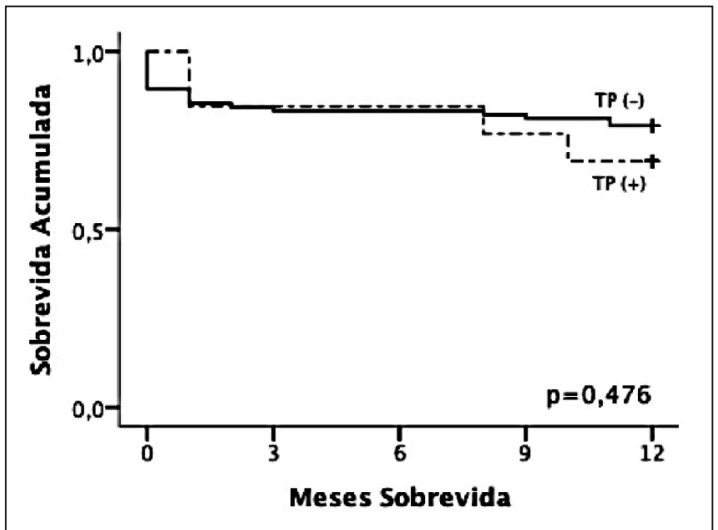

Figura 3. Sobrevida global de pacientes trasplantados con y $\sin$ trombosis portal. 
la anastomosis biliar, que fue tratada exitosamente de manera endoscópica. El último paciente desarrolló una estenosis portal parcial asintomática, que no requirió tratamiento.

La sobrevida global al año de seguimiento fue de $69 \%$ vs un $79 \%$ en los pacientes trasplantados sin TP $(\mathrm{p}=0,476)($ Figura 3).

\section{Discusión}

La causa más frecuente de TP en pacientes con enfermedad hepática avanzada es la cirrosis hepática, siendo ésta responsable de hasta un $30 \%$ de los casos según las diferentes series ${ }^{12,20,21}$. En aquellos pacientes sin cirrosis, los factores trombofílicos locales, adquiridos o congénitos pueden contribuir al desarrollo de una TP21,22.

La patogenia de la TP en hígados cirróticos no se conoce con exactitud. Se la ha relacionado con alteraciones en la arquitectura del hígado y con el aumento de la resistencia vascular, desencadenándose así la hipertensión portal y el enlentecimiento del flujo en el eje porto-esplénico ${ }^{23}$.

La prevalencia de la TP en los pacientes con cirrosis hepática depende de las herramientas diagnósticas utilizadas y de la inclusión o no de los pacientes con hepatocarcinoma (CHC). En los pacientes con CHC, la prevalencia de TP alcanza hasta un $44 \%{ }^{24}$, y aumenta aún más cuando existe cirrosis hepática concurrente. Por esta razón, el diagnóstico de TP en un paciente con cirrosis hepática debe alertar sobre la presencia de un $\mathrm{CHC}^{24}$.

$\mathrm{Si}$ bien existen varios estudios de prevalencia de TP, su incidencia se desconoce con exactitud. Sin embargo, en una cohorte de 251 pacientes con cirrosis hepática enlistados para trasplante hepático, $17(7,4 \%)$ desarrollaron una TP de novo luego de la evaluación inicial en un período de seguimiento de 12 meses $^{25}$.

En nuestra serie, la prevalencia de trombosis portal fue de un 12,4\% (13 TP en 105 pacientes), similar a lo descrito en la literatura internacional ${ }^{12,13}$. El diagnóstico preoperatorio se realizó en el $46,2 \%$ de los casos. Esto se debió al uso rutinario de la ecotomografía abdominal con doppler como método de pesquisa, complementado con resonancia magnética ante la presencia de alteraciones en el flujo portal intra o extrahepático. De acuerdo a lo publicado, esta modalidad diagnóstica permite una detección de TP que varía entre el $26 \%$ y el $87 \%$. Sin embargo, existe una alta tasa de falsos negativos que se explican por la extensión de la TP y por la identificación de colaterales en lugar de la vena porta ${ }^{9}$.

En relación al tratamiento anticoagulante en el preoperatorio, sólo se justifica si las características del trombo son de aparición reciente. Francoz et al ${ }^{25}$, evaluaron la anticoagulación en pacientes con trombosis portal enlistados para trasplante hepático. La proporción de pacientes en que hubo recanalización parcial o completa fue significativamente mayor en aquellos que recibieron anticoagulación (8/19) comparado con los que no recibieron $(0 / 10)$. No hubo complicaciones ni suspensión de tratamiento. Por otra parte, la sobrevida postrasplante fue menor en aquellos pacientes con trombosis completa. Estos datos apoyan el uso de anticoagulación en los pacientes con cirrosis y trombosis portal, enlistados para trasplante. En nuestra serie hubo resolución completa en los tres pacientes que tuvieron tratamiento anticoagulante en la etapa pretrasplante. Dos estudios demostraron tasas de recanalización de 50\% y $75 \%$, respectivamente al emplear heparina de bajo peso molecular, sin efectos secundarios graves, pero con tiempos de administración prolongados ${ }^{26,27}$.

Varias clasificaciones se han descrito para la TP. Sin embargo, una de las ventajas de la clasificación de Yerdel, en concordancia con lo propuesto por Lendoire et $\mathrm{al}^{9}$, es que permite correlacionar el tipo de trombosis con la técnica quirúrgica y el pronóstico del trasplante. El tipo 4 de la clasificación tiene una morbilidad y mortalidad elevada ${ }^{14}$.

El flujo sanguíneo hepático depende hasta un $75 \%$ de la vena porta. Este flujo luego del trasplante debe ser adecuado para que el TH sea exitoso. La reconstrucción portal generalmente se realiza con anastomosis término-terminal entre la vena porta del receptor y el donante. La técnica para restablecer el flujo portal en aquellos pacientes con TP depende de la extensión de la trombosis y la experiencia adquirida por el equipo de trasplante.

Inicialmente la trombosis completa de la vena porta era considerada contraindicación absoluta para el trasplante hasta que los resultados fueron mejorando con la trombectomía y la interposición de injerto entre la confluencia portomesentérica y la vena porta ${ }^{8}$.

En las trombosis grado I y II de la clasificación de Yerdel, se recomienda la trombectomía simple, mientras que en las trombosis grado III debe realizarse una trombectomía más extensa. En nuestra serie, en 7 pacientes fue necesario realizar una trombectomía por eversión ${ }^{28}$.

En 2 de nuestros pacientes fue necesario realizar un puente venoso porto-mesentérico, por trombosis de la confluencia mesentérico portal. En casos en que no se puede realizar una trombectomía de la vena porta y además exista una trombosis de la VMS, puede utilizarse la vena coronaria para asegurar el flujo de entrada ${ }^{10}$.

Uno de los principales riesgos de los pacientes trasplantados con TP es el de retrombosis ${ }^{9}$. En 
nuestra serie no se presentó esta complicación, probablemente por el número reducido de pacientes. En otras series, la incidencia varía entre un 4,2\% y un $38 \%$. Se ha recomendado la anticoagulación profiláctica por un período de tres meses. En nuestra serie, cuatro pacientes recibieron anticoagulación durante seis meses. Se recomienda la realización de doppler portal cada 1-3 días durante las primeras dos semanas postrasplante ${ }^{11}$.

La causa de retrombosis no está clara. Se postula que podría deberse al daño intimal que se produce durante la trombectomía, debido a que estos pacientes presentan una mayor frecuencia de retrombosis que en aquellos pacientes en los que se realiza interposición de vena con paredes sanas ${ }^{11}$.

Nuestra mortalidad fue de un $15,4 \%$, cifra comparable a lo publicado en la literatura internacional $^{9,14}$, indicador de la alta complejidad de estos pacientes.

En relación a la sobrevida obtenida, es concordante con lo reportado en la literatura ${ }^{29}$. En comparación con los pacientes trasplantados sin TP, la diferencia no fue significativamente menor. Estos resultados permiten no descartar del trasplante a pacientes seleccionados, que de otra manera fallecerían en menos de un año.

\section{Referencias}

1. Alqahtani SA. Update in liver transplantation. Curr Opin Gastroenterol. 2012;28:230-8.

2. Merion RM. Current Status and Future of Liver Transplantation. Semin Liver Dis. 2010;30:411-21.

3. Brown KA. Liver transplantation. Curr Opin Gastroenterol. 2005;21:331-6.

4. Merion RM, Schaubel DE, Dykstra DM, Freeman RB, Port FK, Wolfe RA. The survival benefit of liver transplantation. Am J Transplant. 2005;5:307-13.

5. Hepp J, Zapata R, Buckel E, Martínez J, Uribe M, Díaz JC, et al. Trasplante hepático en Chile: Aspectos generales, indicaciones y contraindicaciones (Documento de consenso). Rev Med Chile 2008;136:793-804.

6. Varma V, Mehta N, Kumaran V, Nundy S. Indications and contraindications for liver transplantation. Int $\mathrm{J}$ Hepatol. 2011;2011:121862. Epub 2011 Oct 5.

7. Ahmed A, Keeffe EB. Current indications and contraindications for liver transplantation. Clin Liver Dis. 2007;11:227-47.

8. Manzanet G, Sanjuán F, Orbis P, López R, Moya A, Juan $\mathrm{M}$, et al. Liver transplantation in patients with portal vein thrombosis. Liver Transpl. 2001;7:125-31.

9. Lendoire J, Raffin G, Cejas N, Duek F, Barros Schelotto $\mathrm{P}$, Trigo $\mathrm{P}$, et al. Liver transplantation in adult patients with portal vein thrombosis: risk factors, management and outcome. HPB (Oxford). 2007;9:352-6.

10. Seu P, Shackleton CR, Shaked A, Imagawa DK, Olthoff
KM, Rudich SR, et al. Improved results of liver transplantation in patients with portal vein thrombosis. Arch Surg. 1996;131:840-4.

11. Sharma R, Kashyap R, Jain A, Safadjou S, Graham M, Dwivedi AK, et al. Surgical Complications Following Liver Transplantation in Patients with Portal Vein Thrombosis-A Single-Center Perspective. J Gastrointest Surg. 2010;14:520-7.

12. Ogren M, Bergqvist D, Bjorck M, Acosta S, Eriksson H, Sternby NH. Portal vein thrombosis: prevalence, patient characteristics and lifetime risk: a population study based on 23,796 consecutive autopsies. World J Gastroenterol. 2006;12:2115-9.

13. Nonami T, Yokoyama I, Iwatsuki S, Starzl TE. The incidence of portal vein thrombosis at liver transplantation. Hepatology 1992;16:1195-8.

14. Yerdel MA, Gunson B, Mirza D, Karayalçin K, Olliff S, Buckels J, et al. Portal vein thrombosis in adults undergoing liver transplantation: risk factors, screening, management, and outcome. Transplantation 2000;69:187381.

15. Belghiti J, Panis Y, Sauvanet A, Gayet B, Fedeke F. A new technique of side to side caval anastomosis during orthotopic hepatic transplantation without inferior vena caval occlusion. Surg Gynecol Obstet. 1992;175:270-2.

16. Jones R, Hardy KJ, Fletcher DR, Michell I, McNicol PL, Angus PW. Preservation of the inferior vena cava in orthotopic liver transplantation with selective use of veno-venous bypass: the piggy back operation. Transplant Proc. 1992;24:189-191.

17. Martínez J. Implantación con técnica de Piggy-Back y anastomosis cavo-cava latero-lateral en trasplante hepático ortotópico. Experiencia inicial en un Centro Universitario. Rev Chil Cir. 2004;4:317-31.

18. Dindo D, Demartines N, Clavien PA. Classification of surgical complications: a new proposal with evaluation in a cohort of 6336 patients and results of a survey. Ann Surg. 2004;240:205-13.

19. Clavien PA, Barkun J, de Oliveira ML, Vauthey JN, Dindo D, Schulick RD, et al. The Clavien-Dindo classification of surgical complications: five-year experience. Ann Surg. 2009;250:187-96.

20. Janssen HL. Changing perspectives in portal vein thrombosis. Scand J Gastroenterol Suppl. 2000;232:6973.

21. Janssen HL, Wijnhoud A, Haagsma EB, van Uum SH, van Nieuwkerk CM, Adang RP, et al. Extrahepatic portal vein thrombosis: aetiology and determinants of survival. Gut 2001;49:720-4.

22. Amitrano L, Brancaccio V, Guardascione MA, Margaglione M, Iannaccone L, D'Andrea G, et al. Inherited coagulation disorders in cirrhotic patients with portal vein thrombosis. Hepatology 2000;31:345-8.

23. Villa DC, Condat B. Portal vein thrombosis in adults: Pathophysiology, pathogenesis, and management. J Hepatol. 2000;32:865-71. 
24. Hoekstra J, Janssen HL. Vascular liver disorders (II): portal vein thrombosis. Neth J Med. 2009;67:46-53.

25. Francoz C, Belghiti J, Vilgrain V, Sommacale D, Paradis $\mathrm{V}$, Condat B, et al. Splanchnic vein thrombosis in candidates for liver transplantation: usefulness of screening and anticoagulation. Gut 2005;54:691-7.

26. Senzolo M, Ferronato C, Burra P, Sartori MT. Anticoagulation for portal vein thrombosis in cirrhotic patients should be always considered. Intern Emerg Med. 2009;4:161-2.

27. Amitrano L, Guardascione MA, Menchise A, Martino $\mathrm{R}$, Scaglione M, Giovine S, et al. Safety and Efficacy of
Anticoagulation Therapy With Low Molecular Weight Heparin for Portal Vein Thrombosis in Patients With Liver Cirrhosis. J Clin Gastroenterol. 2010;44:448-51.

28. Dumortier J, Czyglik O, Poncet G, Blanchet MC, Boucaud C, Henry L, et al. Eversion thrombectomy for portal vein thrombosis during liver transplantation. Am J Transplant. 2002;2:934-8.

29. Gimeno FA, Calvo J, Loinaz C, Meneu JC, Perez B, Gomez R, et al. Comparative analysis of the results of orthotopic liver transplantation in patients with and without portal vein thrombosis. Transplant Proc. 2005;37:3899-903. 